Madurai

Tamilnadu 625020, India

Correspondence: $\vee$ Vedantham

Tel: + 914525356100

Fax: + 914522530984

E-mail: drvasumathy@yahoo.com

Eye (2005) 19, 1219-1220. doi:10.1038/sj.eye.6701723; published online 1 October 2004

Sir,

\section{Reply to V Vedantham}

We thank Vasumathy Vedantham for his interest in our paper. In reply to his comments:

1. The British Ophthalmological Surveillance Unit (BOSU) is now well established in the UK to assist in the investigation of clinically important or rare eye conditions such as endophthalmitis. The BOSU-reporting scheme is dependent on voluntary reporting and therefore under-reporting of cases is a potential source of error for incidence calculations. A national registry is ideal for monitoring of postoperative endophthalmitis cases but would require the support of the vast majority of ophthalmologists in the UK or India.

2. The incidence of diabetes in the endophthalmitis cases was $10 \%$. This figure was mentioned in our paper under the heading of cataract surgery details. Comparison of outcome between diabetic and nondiabetic eyes was not analysed in this paper.

3. Pseudomonas was cultured from the aqueous sample but not the vitreous in 3/5 Pseudomonas isolates. This was a surprising result as vitreous is a better culture medium for bacteria than aqueous; ${ }^{1}$ however, cases with a positive aqueous tap and negative vitreous tap have been described. ${ }^{1-2}$

As this study was dependent on individual UK centres providing specific information about endophthalmitis cases, we cannot comment on the vitreous/aqueous sampling technique or sample processing at these centres.

4. In contrast to several Indian endophthalmitis series, ${ }^{3-5}$ no cases of fungi were isolated in our UK survey. Fungal endophthalmitis following cataract surgery has been described as having a prolonged latency period of weeks to months after intraocular inoculation., 5 For our UK survey, we only included cases diagnosed within 6 weeks of cataract surgery, excluding potential delayed onset cases due to fungal infection.
Differences in climate, operating theatre conditions or the sample processing technique may also be important. Information on the antibiotic susceptibility of ocular isolates was not requested for our study.

5. The role of systemic antibiotics in the treatment of endophthalmitis remains unclear. Patients randomised to the intravenous (IV) antibiotic group in the Endophthalmitis Vitrectomy Study (EVS) ${ }^{7}$ received ceftazidime and amikacin (oral ciprofloxacin instead of ceftazidime if the patient was allergic to penicillin). There was no statistically significant difference in outcomes between the IV antibiotic group and the control group. Animal studies have shown inadequate intraocular drug levels of amikacin in inflamed, aphakic, vitrectomized eyes following IV treatment. ${ }^{8}$ IV vancomycin or cefazolin may provide better Gram-positive coverage in aphakic, inflamed eyes than ceftazidime or amikacin..$^{9}$ Therefore, the results of systemic antibiotics in the EVS may not apply to other drug combinations.

\section{References}

1 Beyer TL, Vogler G, Sharma D, O'Donnell FE. Protective barrier effect of the posterior lens capsule in exogenous bacterial endophthalmitis - an experimental primate study. Invest Ophthalmol Vis Sci 1984; 25: 108-112.

2 Barza M, Pavan PR, Doft BH, Wisniewski SR, Wilson LA, Han $\mathrm{DP}$ et al. Evaluation of microbiological diagnostic techniques in postoperative endophthalmitis in the Endophthalmitis Vitrectomy Study. Arch Ophthalmol 1997; 115(9): 1142-1150.

3 Anand AR, Therese KL, Madhavan HN. Spectrum of aetiological agents of postoperative endophthalmitis and antibiotic susceptibility of bacterial isolates. Indian J Ophthalmol 2000; 48: 123-128.

4 Kunimoto DY, Das T, Sharma S, Jalali S, Majji AB, Gopinathan $U$ et al. Microbiologic spectrum and susceptibility of isolates: part I. Postoperative endophthalmitis. Endophthalmitis Research Group. Am J Ophthalmol 1999; 128(2): 240-242.

5 Narang S, Gupta A, Gupta V, Dogra MR, Ram J, Pandav SS et al. Fungal endophthalmitis following cataract surgery: clinical presentation, microbiological spectrum and outcome. Am J Ophthalmol 2001; 132: 609-617.

6 Pflugfelder SC, Flynn HW, Zwickey TA, Forster RK, Tsiligianni A, Culbertson W et al. Exogenous fungal endophthalmitis. Ophthalmology 1988; 95: 19-30.

7 EVS. Results of the Endophthalmitis Vitrectomy Study. A randomized trial of immediate vitrectomy and of intravenous antibiotics for the treatment of postoperative bacterial endophthalmitis. Endophthalmitis Vitrectomy Study Group. Arch Ophthalmol 1995; 113(12): 1479-1496.

8 El-Massry A, Meredith TA, Aguilar HE, Shaarawy A, Kincaid $\mathrm{M}$, Dick J et al. Aminoglycoside levels in the rabbit vitreous cavity after intravenous administration. Am J Ophthalmol 1996; 122: 684-689.

9 Davis JL. Intravenous antibiotics for endophthalmitis. Am J Ophthalmol 1996; 122(5): 724-726. 
S Kamalarajah, G Silvestri, N Sharma, A Khan, B Foot, $\mathrm{R}$ Ling, $\mathrm{G}$ Cran and R Best

Department of Ophthalmology

Altnagelvin Hospital

Londonderry BT 47 6SB, UK

Correspondence: 5 Kamalarajah

Tel: + $442871345171 \times 3776$

Fax: + 442871611218

E-mail: sri17@btinternet.com

Eye (2005) 19, 1220-1221. doi:10.1038/sj.eye.6701726; published online 1 October 2004

Sir,

Retinal haemorrhages following Retcam screening for retinopathy of prematurity

We read with interest the article by Adams $e t a l^{1}$ describing retinal haemorrhages following Retcam examination for retinopathy of prematurity (ROP). On both visits, they detected no retinal haemorrhages initially by Retcam, which were detected later by indirect ophthalmoscopy, although they do not mention the stage of vessel maturation or presence of ROP. We routinely perform ROP screening by the Retcam and have not observed any retinal haemorrhages. Following the authors report, we performed indirect ophthalmoscopy 60 min after ROP screening with Retcam in 50 eyes of 25 children; however, failed to detect such retinal haemorrhages and it seems to be of rare occurrence. It is possible that immature fragile vasculature in very premature babies as in this case or very vascular ROP may present with retinal haemorrhages by inadvertent ocular pressure during the Retcam examination.

A rise in intraocular pressure is not uncommon as disc pulsations are induced during examination when pressure is applied from the hand piece. Although we use the second-generation 130-degree ROP lens, the presence of small pupils and persistent ocular movement makes it difficult to visualize the periphery; with a need to tilt the head and the hand piece in various configurations to obtain a suitable view, which causes an increase in pressure. It is essential to ensure that the coupling solution is replenished repeatedly as it flows out of the eye during the examination, as a lack of it causes a blurring of image, with more manoeuvers by the observer. Proper immobilization of the head is essential to prevent sudden head jerks and consequent injury.

With modern neonatal care as younger preterm infants survive, such vascular incidents may be more common.
Although such cases are rare, this report guides us to take utmost care during Retcam examination.

\section{Acknowledgements}

Financial interest: None.

\section{Reference}

1 Adams GG, Clark BJ, Fang S, Hill M. Retinal haemorrhages in an infant following RetCam screening for retinopathy of prematurity. Eye 2004; 18(6): 652-653.

RV Azad, P Chandra, N Pal and DV Singh

Vitreo-retina Services

Dr Rajendra Prasad Centre for Ophthalmic Sciences

All India Institute of Medical Sciences

Ansari Nagar

New Delhi 110029, India

Correspondence: RV Azad

Tel: + 911126293187

Fax: +911126852919

E-mail: rajvardhanazad@hotmail.com

Eye (2005) 19, 1221. doi:10.1038/sj.eye.6701724;

published online 1 October 2004

Sir,

\section{Reply to RV Azad et al}

We thank Professor Azad and colleagues for their interest in our case report.

At the time of examinations the baby was between 32 and 34 weeks gestation, with no suggestion of abnormalities of retinal vascularisation.

We concur with their view that this is a rare occurrence that we noted during an audit of RetCam screening against conventional indirect ophthalmoscopy. At the time of these events, the manufacturers considered that some 1 million RetCam examinations had taken place with no other similar report. They were not aware of any other Unit undertaking a similar audit process, and we are therefore interested to know that Professor Azad and colleagues have not demonstrated a similar occurrence in their study.

We agree with Professor Azad and colleagues that care must be taken not to apply excessive pressure on the eye when using the RetCam. We advise that all neonatal screening should use the lighter ROP screening head and not the heavier standard 